\title{
In Situ Transmission Electron Microscopic Investigation of Coalescence Dynamics of Au Nanoparticles Embedded in Solid Potassium Bromide
}

\author{
Wenbo $\mathrm{Xin}^{1 *}$
}

\author{
1. Department of Materials Science and Engineering, University of California - Los Angeles, 410 \\ Westwood Plaza, Los Angeles, CA 90095 \\ * Corresponding author: kevin.xwsu@ gmail.com
}

Understanding dynamic behaviors of nanoparticles under different circumstances is of essential importance to tune their sustained functionalities in any given application [1-3]. Thanks to the advances in the in-situ transmission electron microscopy (TEM), coalescence dynamics of Au nanoparticles (AuNPs) in aqueous solution have been extensively investigated [4,5]. However, there is rare attention on the study of these activities in the presence of solid surfactants, such as potassium bromide (KBr).

Our group recently reports $\mathrm{Br}$ ion $\left(\mathrm{Br}^{-}\right)$, serving as the surfactant, can direct the anisotropic growth of gold nanocrystals [6,7]. It is thus of interest to devise experiments to explore detailed interactions of $\mathrm{KBr}$, the provider of $\mathrm{Br}^{-}$, with gold clusters/nanoparticles. In this report, we demonstrate a novel approach to prepare AuNPs in solid KBr environment. We monitor the coalescence kinetics of AuNPs with $\mathrm{KBr}$ and reveal that $\mathrm{KBr}$ has dominant effects in the fusion process of AuNPs.

As is known, halogen salts such as $\mathrm{NaCl}$ and $\mathrm{KBr}$ are vulnerable to high-energy electron beams [8]. It is, therefore, challenging to monitor their activities in TEM in terms of a long period (e.g. more than a few seconds). Here, we employed graphene sheet as the support for both KBr and AuNPs. Figure 1 (a) shows a representative TEM image of AuNPs associating with $\mathrm{KBr}$ on the graphene substrate. Interfaces of solid $\mathrm{KBr}$ and AuNPs are highlighted in the red box, demonstrating nanoparticles are entirely surrounded by $\mathrm{KBr}$ crystallites. The higher magnification image in Figure 1(b) clearly illustrates a typical (100)-orientated crystalline structure. The fast Fourier transformation (FFT) of highlighted area is presented in the inset of Figure 1(b). The lattice distance is measured as $3.3 \AA$, corresponding to $\mathrm{d}_{\text {spacing }}$ of $\mathrm{KBr}(200)$. It is surprising to observe $\mathrm{KBr}$ crystallites preserve certain stability against the high energy electron beam (dose rate of $1.6 \times 10^{5} \mathrm{e}^{-} / \AA^{2} \cdot \mathrm{s}$ at $300 \mathrm{keV}$ ). It has been reported graphene has the capability of stabilizing nanocrystals such as gold nanowires [9] and the square ice [10]. We will discuss the mechanism of graphene-stabilized $\mathrm{KBr}$ under the electron irradiation.

We then investigate the electron bean induced coalescence of AuNPs in the presence of KBr. Figures 2 (a)-(e) are time-lapsed TEM images of the fusion process from $10 \mathrm{~s}$ to $87 \mathrm{~s}$. Overall, coalesced nanoparticles get contacted, form the neck, and complete the fusion when the neck area is filled up. In this study, three nanoparticles finally evolve to a Au nanorod at $87 \mathrm{~s}$. Neck growth, which is driven by surface energy minimization and realized by surface atom diffusion, characterizes the coalescence kinetics [11]. We record the growth rate of the neck between particle 1 and 2 and summarize the relation (neck width $\boldsymbol{D}$ versus time $\boldsymbol{t}, \boldsymbol{D} \sim \boldsymbol{t}$ ) in Figure 2 (b). It is of interest to note two obvious differences in this relation from AuNPs coalesced with and without $\mathrm{KBr}$. First, the neck grows significantly faster associated with $\mathrm{KBr}$. Second, two growth stages exist in the case of AuNPs with this capping agent. We will extend our discussions to the dynamic state of $\mathrm{KBr}$ under electron irradiation and provide plausible explanations to these impressive phenomena. This work will enhance the understanding of the role of solid surfactants in directing the growth of nanocrystals. 
References:

[1] H.-G. Liao et al., Science 345 (2014), p. 916.

[2] D. Li et al., Science 336 (2012), p. 1014.

[3] J. H. Park et al., Nano Letter 15 (2015), p. 5314.

[4] W. Xin et al., Journal of Physical Chemistry C 123 (2019), p. 4523.

[5] C. Zhu et al., Nature Communication 9, 421 (2018).

[6] W. Xin et al., Nano Letter 18 (2018), p. 1875.

[7] W. Xin et al., ACS Applied Materials Interfaces 9 (2017), p. 6246.

[8] W. Zhu et al., Nature 528 (2015), p. E1.

[9] W. Xin et al., Chemical Communication 54 (2018), p. 4124

[10] G. Algara-Siller et al., Nature 519 (2015), p. 443.

[11] M.A. Asoro et al., Acta Materialia 81 (2014), p. 173.

[12] The author acknowledges funding from the U.S. department of defense (fund \# 000-16-C-0081).
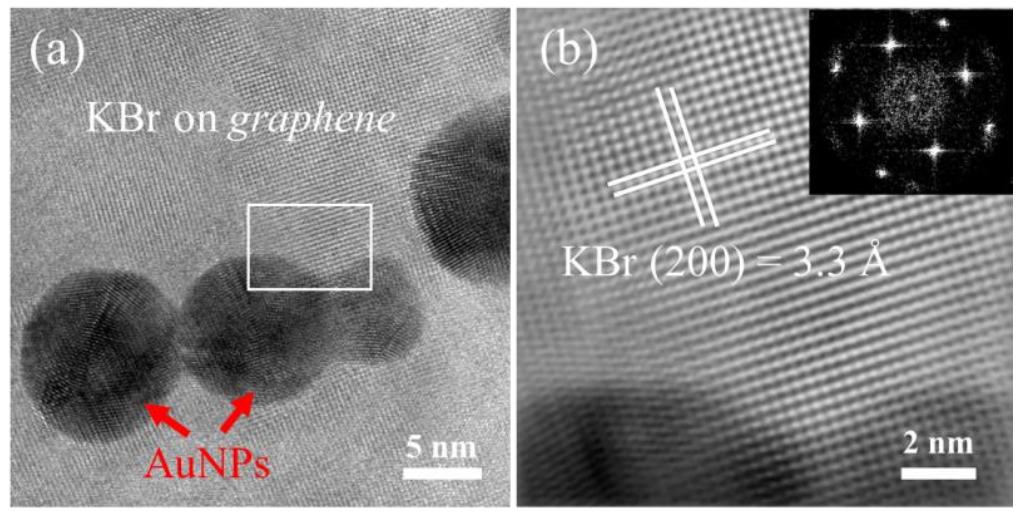

Figure 1. (a) A representative TEM image of Au nanoparticles embeded in solid KBr crystallites. (b) High resolution image of the interface between $\mathrm{Au}$ nanoparticles and $\mathrm{KBr}$. Crystalline structure of $\mathrm{KBr}$ is clearly identified.
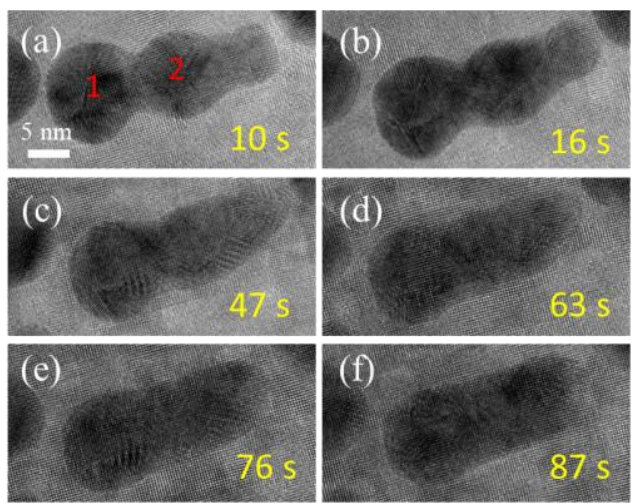

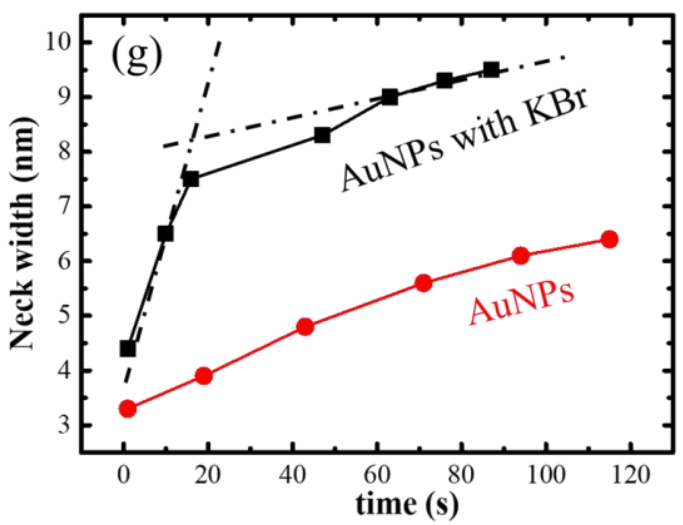

Figure 2. Coalescence kinetics of Au nanoparticles capped with KBr. (a)-(f) Time-lapsed TEM images of the coalescence process. (g) Neck growth over time between particle 1 and 2. For comparison, the neck growth of AuNPs free of $\mathrm{KBr}$ is also provided (red curve). 